\title{
TU/e EmonOWEN

\section{Measurement of shock-induced guided and surface acoustic waves along boreholes in poroelastic materials}

\section{Citation for published version (APA):}

Chao, G. E., Smeulders, D. M. J., \& Dongen, van, M. E. H. (2006). Measurement of shock-induced guided and surface acoustic waves along boreholes in poroelastic materials. Journal of Applied Physics, 99(9), 094904-1/7. [094904]. https://doi.org/10.1063/1.2191467

DOI:

10.1063/1.2191467

Document status and date:

Published: 01/01/2006

\section{Document Version:}

Publisher's PDF, also known as Version of Record (includes final page, issue and volume numbers)

\section{Please check the document version of this publication:}

- A submitted manuscript is the version of the article upon submission and before peer-review. There can be important differences between the submitted version and the official published version of record. People interested in the research are advised to contact the author for the final version of the publication, or visit the $\mathrm{DOI}$ to the publisher's website.

- The final author version and the galley proof are versions of the publication after peer review.

- The final published version features the final layout of the paper including the volume, issue and page numbers.

Link to publication

\section{General rights}

Copyright and moral rights for the publications made accessible in the public portal are retained by the authors and/or other copyright owners and it is a condition of accessing publications that users recognise and abide by the legal requirements associated with these rights.

- Users may download and print one copy of any publication from the public portal for the purpose of private study or research.

- You may not further distribute the material or use it for any profit-making activity or commercial gain

- You may freely distribute the URL identifying the publication in the public portal.

If the publication is distributed under the terms of Article 25fa of the Dutch Copyright Act, indicated by the "Taverne" license above, please follow below link for the End User Agreement:

www.tue.nl/taverne

Take down policy

If you believe that this document breaches copyright please contact us at:

openaccess@tue.nl

providing details and we will investigate your claim. 


\title{
Measurements of shock-induced guided and surface acoustic waves along boreholes in poroelastic materials
}

\author{
Gabriel $\mathrm{Chao}^{\mathrm{a})}$ \\ Department of Geotechnology, Delft University of Technology, P.O. Box 5028, 2600 GA Delft, The \\ Netherlands and Department of Applied Physics, Eindhoven University of Technology, P.O. Box 513, 5600 \\ $M B$ Eindhoven, The Netherlands \\ D. M. J. Smeulders \\ Department of Geotechnology, Delft University of Technology, P.O. Box 5028, 2600 GA Delft, \\ The Netherlands \\ M. E. H. van Dongen \\ Department of Applied Physics, Eindhoven University of Technology, P.O. Box 513, 5600 MB Eindhoven, \\ The Netherlands
}

(Received 6 October 2005; accepted 4 March 2006; published online 11 May 2006)

\begin{abstract}
Acoustic experiments on the propagation of guided waves along water-filled boreholes in water-saturated porous materials are reported. The experiments were conducted using a shock tube technique. An acoustic funnel structure was placed inside the tube just above the sample in order to enhance the excitation of the surface modes. A fast Fourier transform-Prony-spectral ratio method is implemented to transform the data from the time-space domain to the frequency-wave-number domain. Frequency-dependent phase velocities and attenuation coefficients were measured using this technique. The results for a Berea sandstone material show a clear excitation of the fundamental surface mode, the pseudo-Stoneley wave. The comparison of the experimental results with numerical predictions based on Biot's theory of poromechanics [J. Acoust. Soc. Am. 28, 168 (1956)], shows that the oscillating fluid flow at the borehole wall is the dominant loss mechanism governing the pseudo-Stoneley wave and it is properly described by the Biot's model at frequencies below $40 \mathrm{kHz}$. At higher frequencies, a systematic underestimation of the theoretical predictions is found, which can be attributed to the existence of other losses mechanisms neglected in the Biot formulation. Higher-order guided modes associated with the compressional wave in the porous formation and the cylindrical geometry of the shock tube were excited, and detailed information was obtained on the frequency-dependent phase velocity and attenuation in highly porous and permeable materials. The measured attenuation of the guided wave associated with the compressional wave reveals the presence of regular oscillatory patterns that can be attributed to radial resonances. This oscillatory behavior is also numerically predicted, although the measured attenuation values are one order of magnitude higher than the corresponding theoretical values. The phase velocities of the higher-order modes are generally well predicted by theory. (C) 2006 American Institute of Physics. [DOI: $10.1063 / 1.2191467]$
\end{abstract}

\section{INTRODUCTION}

Surface elastic-acoustic waves are common phenomena in nature and widely used in technological applications. Their study covers a broad band of frequencies and wavelengths, ranging from surface seismic waves in the infrasound domain to laser-generated surface acoustic waves at ultrasound frequencies. The wide spectrum of applications includes the inversion of surface wave data for subsurface imaging, design of filters for interdigital transducers, material characterization, and recently, the imaging of elastic properties of bones.

Research on the characterization of porous materials by acoustics means has been carried out by Fellah et al. ${ }^{1}$ and Fan $e t a .^{2}$ among others. In this respect, there has been an increasing interest in the study of surface waves along poroelastic media in the last years. Allard et al. ${ }^{3,4}$ reported in-

${ }^{a)}$ Electronic mail: g.e.chao@tue.nl vestigations on laser-irradiated surface waves along porous materials. The analysis of the surface deformation caused by the surface modes provides information on the mechanical properties of the porous solid, particularly the shear modulus. ${ }^{5}$ These series of experiments were performed in plane interfaces between a fluid (air or water) and a fully saturated (gas or liquid) porous material. However, in many applications, for instance related to oil exploration, the interface is cylindrical. In this case, theoretical and experimental investigations of acoustic wave propagation along boreholes surrounded by poroelastic formations have provided promising evidence about the dependence of the permeability and porosity of the reservoir on the frequency-dependent velocities and attenuations of the surface modes. ${ }^{6-11}$ Nevertheless, there is a lack of a full understanding of the underlying physical mechanisms which govern the propagation of the surface waves. Even for the body waves, there is a long way before the diverse loss mechanisms induced due to the pas- 
sage of an elastic wave in a porous medium can be completely understood. These dissipative processes are highly scale dependent and can be in principle classified according to the ratio between the wavelength and the size of the inhomogeneities of the porous medium. ${ }^{12}$ If the wavelength is much larger than the pore size, the main source of dissipation is the viscous friction due to the relative motion between the solid and the fluid phase. This mechanism can be properly described by the Biot theory. ${ }^{13-15}$ One of the basic assumptions of the Biot model is related to the homogeneity of the solid phase. Heterogeneities in the composites cause waveinduced flow losses due to local compressibility changes which are not considered in the original Biot formulation. These so-called mesoscopic loss mechanisms are of significant importance when the wavelength becomes of the order of magnitude of the size of the heterogeneities. Recently, Pride and Berryman ${ }^{16,17}$ have developed from first principles a set of governing equations for double-porosity dualpermeability materials. These heterogeneous materials are constituted by two distinct porous materials with different lithological and poromechanical properties. At even higher frequencies, when the wavelength is comparable to the grain size, microscopic losses, namely, local flow effects and scattering have to be taken into account.

An accurate determination of the frequency-dependent attenuation under well-defined laboratory conditions will provide useful insight in the frequency-dependent loss mechanisms mentioned above. Furthermore, it constitutes a valuable tool in order to validate the theoretical models, particularly those describing surface wave propagation along boreholes. For this last configuration, the scarce experimental data available is restricted to relatively high frequencies. ${ }^{8,11}$ In this work, a modified shock wave technique is applied to study the propagation of borehole modes in poroelastic formations. One of the advantages of this method is the broad band of frequencies involved in the experiments $(0.5-160 \mathrm{kHz})$. The aim was to obtain accurate frequency-dependent data of the phase velocities and attenuation coefficients of the surface waves, especially in the lowfrequency range. These results are compared with numerical calculations based on Biot's theory in order to assert the validity of the Biot loss mechanisms to describe the attenuation of the surface waves.

The shock tube technique has been utilized in the past to study bulk acoustic wave propagation in fully saturated porous media, ${ }^{18,19}$ in partially saturated porous media, ${ }^{20}$ and borehole modes in poroelastic formations. ${ }^{11}$ This last investigation revealed a quantitative agreement between the theoretical predictions based on Biot's theory and the experimental data for the frequency-dependent phase velocity. However, the attenuation data showed a strong scatter and only a qualitative agreement was found. In this paper we report a modification of the experimental setup that aimed to enhance the excitation of the surface modes and to improve the quality of the attenuation data.

The paper is organized as follows: in Sec. II the different axisymmetric guided modes that may propagate in the experimental configuration are computed and analyzed. In Sec. III the experimental method is described followed by the

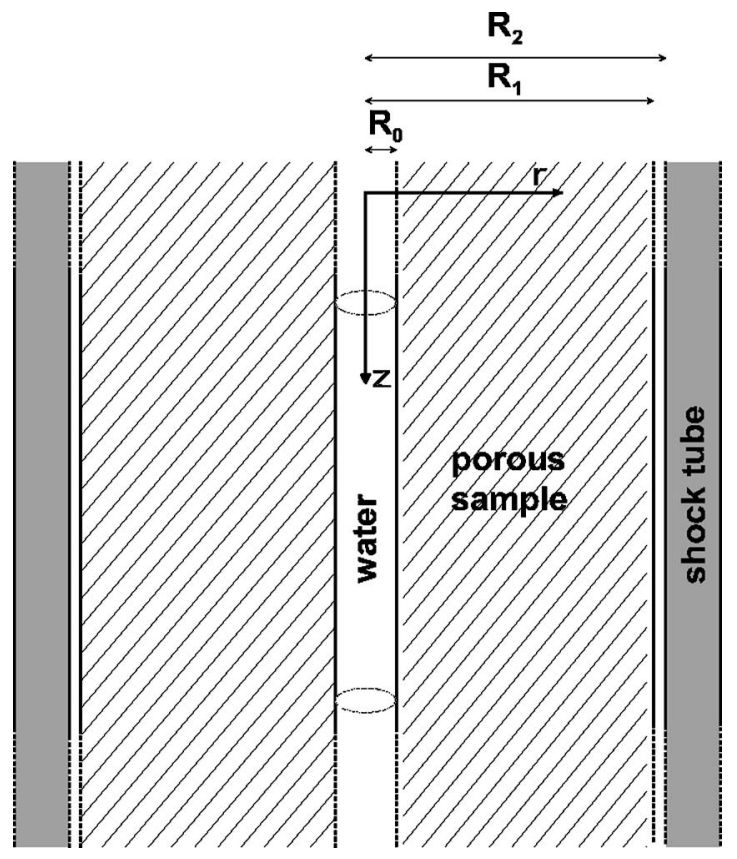

FIG. 1. Experimental configuration: A concentrically drilled porous sample bounded by a cylindrical shell. $R_{0}$ and $R_{1}$ represents the inner and external radius of the sample (see Table $\mathrm{I}$ ), $R_{2}$ is the inner radius of the shock tube $\left(R_{2}=38.5 \mathrm{~mm}\right)$.

experimental results in Sec. IV. The comparison of the measured data with the theoretical predictions allow us to draw the conclusions in Sec. V.

\section{AXISYMMETRIC GUIDED MODES IN POROELASTIC MEDIA}

There is an infinite number of wave modes that can propagate along a cylindrical interface between a liquid and an elastic or poroelastic medium. An extensive study of the different modes for an elastic formation is given by Sinha and Asvadurov. ${ }^{21}$ The modes can preliminary be classified according to their axial symmetry. Axisymmetric modes propagating along an elastic formation include the Stoneley wave and an infinite number of pseudo-Rayleigh waves. Higher nonaxisymmetric sets of borehole modes comprise the flexural modes and the quadrupole modes. We only consider the axisymmetric case where there is no dependence of the displacements and stresses on the azimuthal angle. The configuration is depicted in Fig. 1. The sample consists of a concentric borehole within a porous cylinder and is fully saturated with water. It is placed in the test section of a vertical shock tube. The mathematical procedure to obtain the frequency-dependent wave velocities, attenuation coefficients, displacement, and pore pressure of the guided waves has been described in detail in a previous paper. ${ }^{11}$ In short, the different bulk modes are described through a displacement potential formulation based on Biot's theory. Subsequently, the application of the boundary conditions leads to the dispersion relation, which is solved numerically by means of a zero-search routine in the complex plane. It is important to recall in order to avoid confusion that in the liquid/poroelastic case, the classical Stoneley wave that ex- 


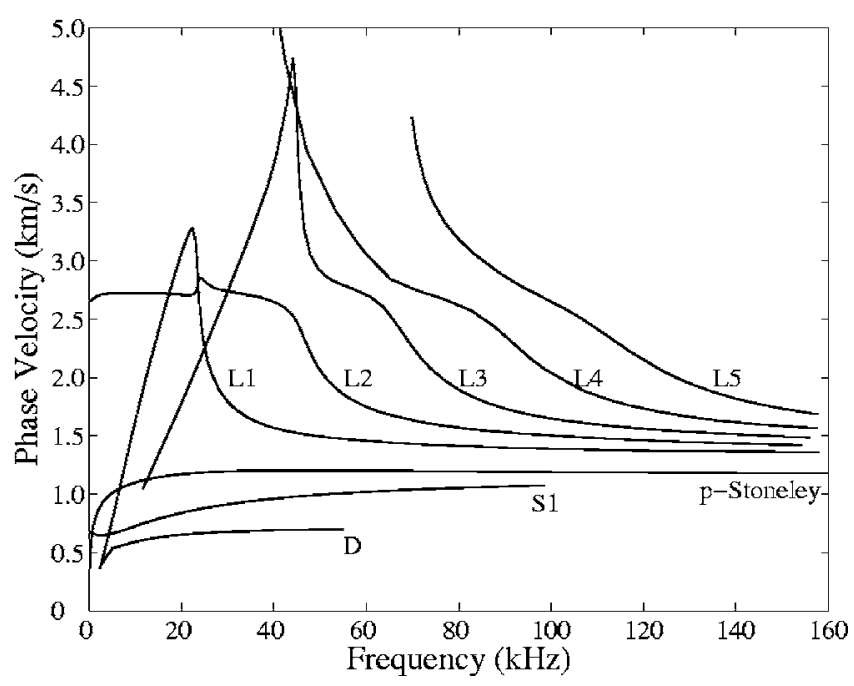

FIG. 2. Experimental configuration: A concentrically drilled porous sample bounded by a cylindrical shell. $R_{0}$ and $R_{1}$ represents the inner and external radius of the sample (see Table I), $R_{2}$ is the inner radius of the shock tube $\left(R_{2}=38.5 \mathrm{~mm}\right)$.

ists in a liquid/elastic interface becomes a pseudo- or leaky wave due to radiation into the Biot slow compressional wave. $^{22}$

Figure 2 shows the guided modes for a Berea sandstone sample. In addition to the pseudo-Stoneley wave which has been extensively studied in previous papers, 8,11 several other modes are identified. The set of $L$ modes is also encountered in liquid-filled pipes ${ }^{23,24}$ and in water-saturated porous cylinders bounded by a cylindrical shell. ${ }^{25}$ In our configuration the first three $L$ modes are propagative at all frequencies. The L2 mode is related to the fast compressional wave at low frequencies. The higher-order $L$ modes are characterized by cutoff frequencies quite similar to the pseudo-Rayleigh waves in a radially infinite poroelastic medium or the undamped modes in a liquid-filled cylindrical cavity. There is one guided mode, the $D$ mode, which is associated with the slow compressional wave in the porous sample. An extra surface mode, S1, is also present; this mode is a surface mode propagating at the interface between the outer surface of the cylinder and the water gap. This can be inferred from the radial dependence of the average radial displacement and the pore pressure induced by the $\mathrm{S} 1$ mode at representative frequencies of 30,60 , and $90 \mathrm{kHz}$. The average radial displacement is defined as follows: $\zeta=(1-\phi) u_{r}+\phi U_{r}$, where $\phi$ is the porosity, $u_{r}$ the radial matrix displacement, and $U_{r}$ the

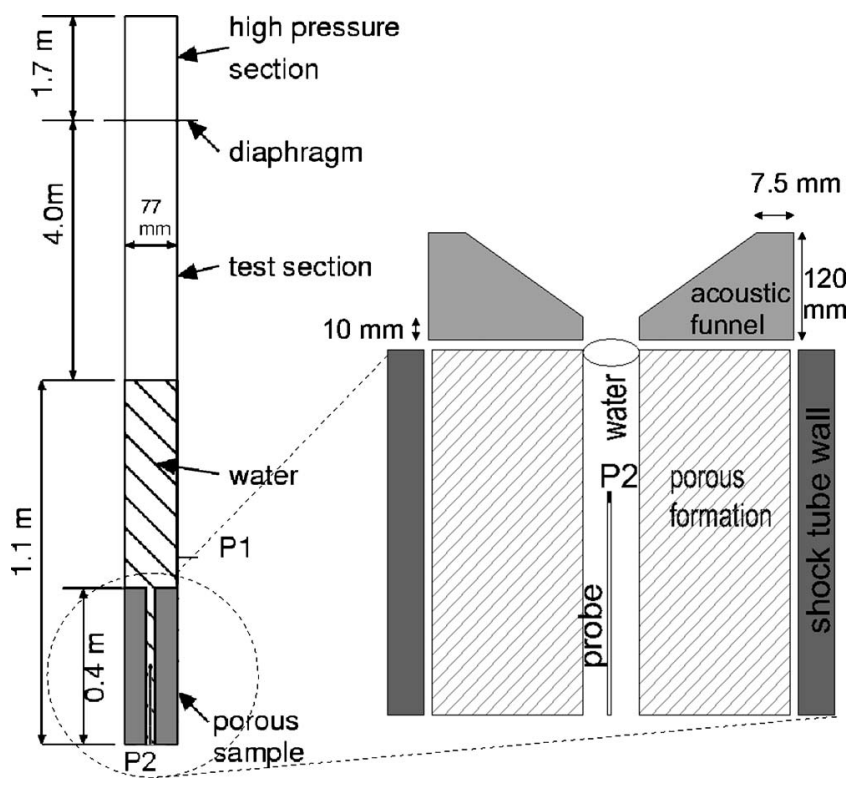

FIG. 4. The vertical shock tube employed in the acoustic experiments. The measuring section is shown in detail. The drawing is schematic and not to scale.

radial fluid displacement. Inspection of Fig. 3 confirms the assumption about the nature of the $\mathrm{S} 1$ mode. The radial profiles of the average radial displacement and pore pressure depict the characteristic decay into the porous reservoir correlated to a surface wave whose energy is confined to the interface between the outer surface of the porous sample and the water annulus.

\section{EXPERIMENTAL SETUP AND METHODS}

A schematic drawing of the vertical shock tube employed to perform the acoustics experiments is shown in Fig. 4. The vertical shock tube is made out of steel. The length of the tube is approximately $6.8 \mathrm{~m}$ and it has an inner diameter of $77 \mathrm{~mm}$. The shock tube consists of two sections: the highpressure section and the test section which are separated by a plastic diaphragm. The porous sample which contains a concentric borehole is placed at the bottom of the test section and is carefully saturated with water. The water level is approximately $1 \mathrm{~m}$ above the bottom plate of the test section. The lengths of the samples vary from 25 to $40 \mathrm{~cm}$. Previous experiments were reported before, ${ }^{11}$ but it was found that the quality of the attenuation data of the surface waves was ham-

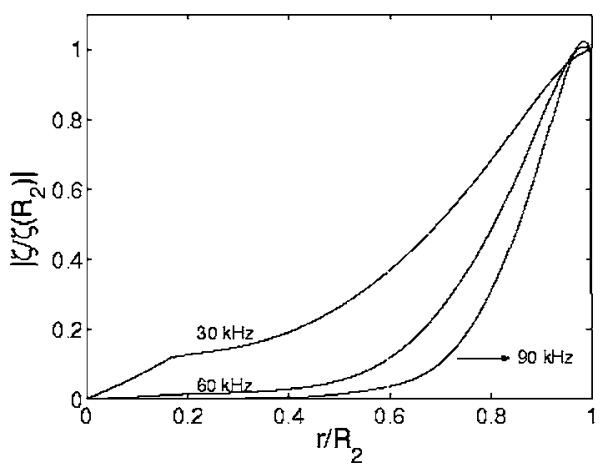

(a)

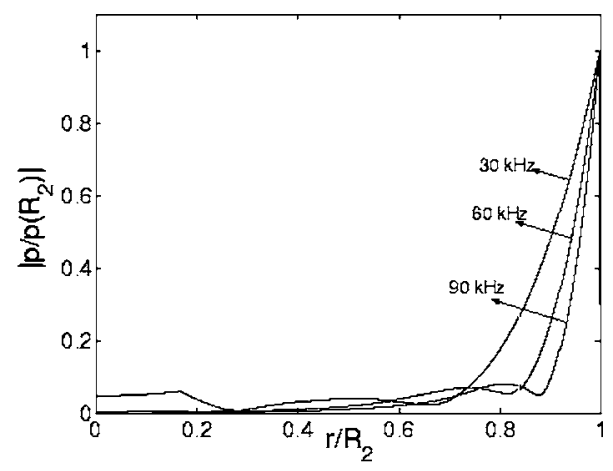

(b)
FIG. 3. Radial dependence of the average radial displacement (a) and pore pressure (b) associated with the S1 mode in the confined reservoir at frequencies of 30,60 , and $90 \mathrm{kHz} . R_{2}$ $=38.5 \mathrm{~mm}$. 
pered by the strong generation of the compressional bulk mode in the porous material, which result in a low signal-tonoise ratio for the surface modes. In order to enhance the excitation of the surface waves and minimize bulk wave generation, an acoustic funnel was placed inside the tube. This structure was designed to shield the top of the sample from the direct impact of the incident wave, and to channel most of the energy into the borehole, thereby strongly reducing the compressional body wave. The funnel structure is screwed to the shock tube wall and separated from the top of the sample by a distance of approximately $1 \mathrm{~mm}$ in order to avoid mechanical contact. The variable cross section was designed to prevent resonances inside this device.

A typical experiment proceeds as follows: The highpressure section is pressurized up to 4 bars with respect to the test section which is at atmospheric pressure. Then the plastic membrane is ruptured. In this way a weak shock wave is generated which travels downwards. It is partially transmitted and partially reflected at the air-water interface. The transmitted wave becomes a steplike acoustic wave which is focused into the borehole by means of the funnel. The pressure inside the borehole is measured by means of a pressure transducer P2 (Druck PCR 81) which is mounted on a mobile probe that can be displaced along the borehole axis. In this way the pressure at different positions can be recorded. Due to the high reproducibility of the experiments these recordings can be combined into a single array of measurements consisting of 25 different pressure signals. The only source of discrepancy between the different experiments is a slight variation of the amplitude of the incident wave in the water, which is due to small fluctuations of the strength of the impinging weak shock wave. This amplitude variation is on the order of $1 \%$ at most. In order to correct for this variation, the measured pressure in the borehole is normalized with respect to the amplitude of the incident wave recorded at the transducer P1 (Kistler 603B) which is located above the funnel. This transducer is also used to trigger the data acquisition system, a high-frequency wave-form recorder (Lecroy 6810, sample rate: $5 \mathrm{MHz}$ ).

Three porous samples were used in the experiments, a natural Berea sandstone sample and two synthetic samples denoted N1 and N2. The synthetic samples are made of sintered crushed glass and have a relatively high permeability in comparison with ordinary rocks. In this way we cover a
TABLE I. Physical properties of the porous formations. N1 and N2 are synthetic porous materials made out of sintered glass, the Berea sandstone is a natural formation. The parameters reported in this table were independently measured in laboratory tests.

\begin{tabular}{lccc}
\hline \hline & Berea sandstone & $\mathrm{N} 1$ & $\mathrm{~N} 2$ \\
\hline Solid density $\rho_{s}\left(\mathrm{~kg} / \mathrm{m}^{3}\right)$ & 2644 & 2590 & 2539 \\
Porosity $\phi$ & 0.20 & 0.53 & 0.465 \\
Permeability $k_{0}(\mathrm{D})$ & 0.36 & 2.9 & 10.8 \\
Tortuosity $\alpha_{\infty}$ & 2.4 & 1.7 & 1.93 \\
Frame bulk modulus $K_{b}(\mathrm{GPa})$ & 10.37 & 1.97 & 2.53 \\
Shear modulus $N(\mathrm{GPa})$ & 4.02 & 1.3 & 2.9 \\
Solid grain bulk modulus $K_{s}(\mathrm{GPa})$ & 36.5 & 36.5 & 36.5 \\
Liquid density $\rho_{f}\left(\mathrm{~kg} / \mathrm{m}^{3}\right)$ & 1000 & 1000 & 1000 \\
Liquid viscosity $\eta(\mathrm{mPa} \mathrm{s})$ & 1.0 & 1.0 & 1.0 \\
Liquid bulk modulus $K_{f}(\mathrm{GPa})$ & 2.25 & 2.25 & 2.25 \\
Dry compressional velocity $v_{p}(\mathrm{~m} / \mathrm{s})$ & 2727 & 1744 & 2170 \\
Dry shear velocity $v_{s}(\mathrm{~m} / \mathrm{s})$ & 1379 & 1033 & 1461 \\
Inner radius $R_{0}(\mathrm{~mm})$ & 6.3 & 6.3 & 6.3 \\
External radius $R_{1}(\mathrm{~mm})$ & 37.1 & 38.4 & 38.4 \\
\hline \hline
\end{tabular}

range of permeability values from $360 \mathrm{mD}$ to $10.8 \mathrm{D}$. The properties of the samples are given in Table I.

In order to obtain the frequency-dependent phase velocities and specific attenuations, the pressure signals are transformed from the space-time domain to the frequency-wavenumber domain. This is performed using a combined fast Fourier transform (FFT)-Prony-spectral ratio technique. This method uses the output for the frequency-dependent phase velocities from the conventional FFT-Prony method, ${ }^{26}$ as the input for the spectral ratio technique. ${ }^{27}$ This extra step significantly improves the determination of the specific attenuation $Q^{-1}$. It is worthwhile to mention that the FFT-Pronyspectral ratio technique can be implemented only when the time signal consists of a single wave mode, which explains the importance of achieving a clear isolation and resolution of the pseudo-Stoneley wave in the time domain by means of the acoustical funnel.

\section{EXPERIMENTAL RESULTS}

In this section the experimental findings are summarized. The first topic to be addressed is the influence on the transmitted waves in the borehole of the enforced energy canalization. A common problem encountered in our previous ex-

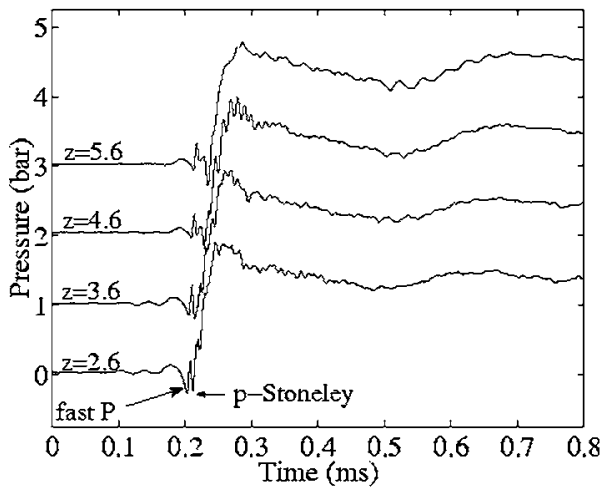

(a)

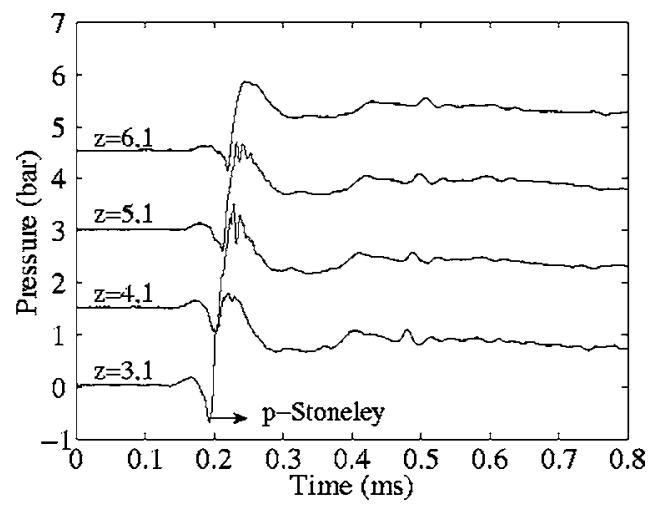

(b)
FIG. 5. Pressure recordings at different depths (b). The position of the pressure transducer $(z)$ is measured in centimeter from the top of the sample. For readability, traces have been shifted upwards. Previous measurements are reported for comparison (a). The time scale is relative to each series of experiments. The arrows indicate the arrival of the high-frequency content of each wave. 


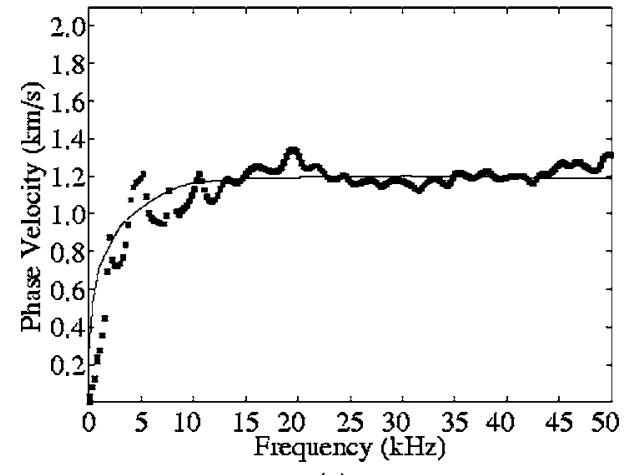

(a)

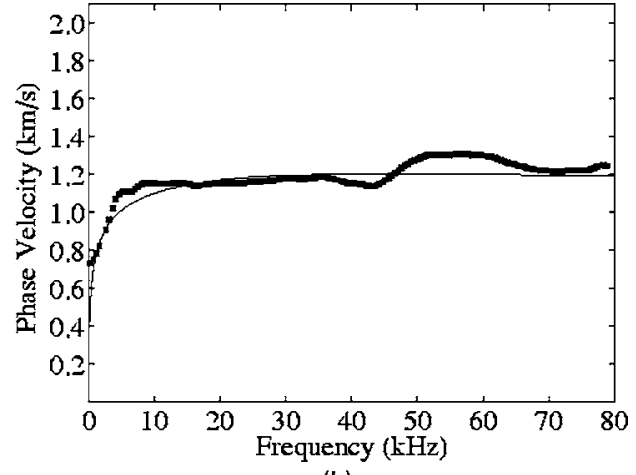

(b)
FIG. 6. Frequency-dependent phase velocity of the pseudo-Stoneley wave in the Berea sample. The experimental results are shown in dots and the theoretical calculations based on Biot's theory as solid lines. The results obtained in the modified shock tube configuration are depicted in (b). For comparison, previous measurements are shown (a). periments in the Berea sandstone sample was the excitation of the guided mode associated with the fast $P$ wave, ${ }^{11}$ as it is shown in Fig. 5(a). This mode overlapped with the pseudoStoneley wave. This overlapping hindered the propagation of the pseudo-Stoneley wave and complicated the analysis. The aim of the modification of the shock tube was to suppress the excitation of the fast compressional wave, which results in a better resolution of the pseudo-Stoneley wave in the recordings. Figure 5(b) shows the measured pressure as a function of time for representative experiments performed in the current experimental configuration. In these series of experiments only one clear arrival is observed. The application of the FFT-Prony method in the complete series of measurements revealed that the wave form is constituted by only one wave mode, the pseudo-Stoneley wave, as shown in Fig. 6(b). An excellent agreement is obtained between the experimental data and the theoretical predictions for the frequencydependent phase velocity. We remark that we were able to measure at frequencies as low as $750 \mathrm{~Hz}$. This is a significant improvement with respect to the previous measurements [Fig. 6(a)], where a strong oscillatory pattern was observed at frequencies below $10 \mathrm{kHz}$.

Next, the FFT-Prony-spectral ratio method was implemented to process the attenuation data. The results in terms of the specific attenuation coefficient $Q^{-1}$ are depicted in Fig. 7(b). Now there is also a very good agreement between theory and experiments for $Q^{-1}$. In Fig. 7(a) the previous attenuation data in terms of the damping coefficient is shown. As a general conclusion we state that regarding the pseudo-Stoneley wave, the quality of the experimental data has improved considerably due to the suppression of the fast compressional wave which to a certain extent overlapped the surface wave in the previous series of experiments.

The theoretical predictions for $Q^{-1}$ slightly underestimate the attenuation measured, particularly at high frequencies $(>40 \mathrm{kHz})$. The shock wave experiments show that the oscillating viscous flow at the borehole wall, which is the predominant dissipative mechanism for the pseudo-Stoneley wave is properly described by the Biot theory for frequencies lower than $40 \mathrm{kHz}$. At frequencies higher than $40 \mathrm{kHz}$, the systematic underestimation of the theory indicates the existence of other loss mechanisms. At high frequencies, where the wavelength becomes of the order of the heterogeneities in the porous material, local compressibility effects, squirt flow losses, and scattering effects, which are not included in the Biot theory, become a significant source of dissipation for the bulk modes. Our results indicate that these mechanisms have some influence on the pseudo-Stoneley wave as well.

Figure 8 shows the frequency-dependent phase velocities of the shock-induced guided waves excited in the experiments performed on the N1 sample. Two modes are distinctly observed, the pseudo-Stoneley wave and the L3 mode. The speed of the pseudo-Stoneley wave is correctly predicted by the numerical computations although fairly higher values are measured at high frequencies. We report experimental data for the pseudo-Stoneley wave at frequencies as low as $3 \mathrm{kHz}$. The L3 mode is related to the curvature of the geometry and cannot be directly associated with any of the surface modes propagating along the borehole wall. We have found an excellent agreement for the phase velocity between theory and experiments for this mode even close to the cutoff frequency. However, since it was not possible to

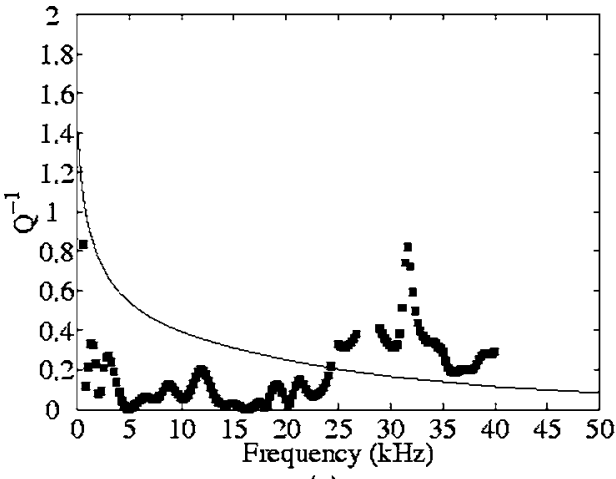

(a)

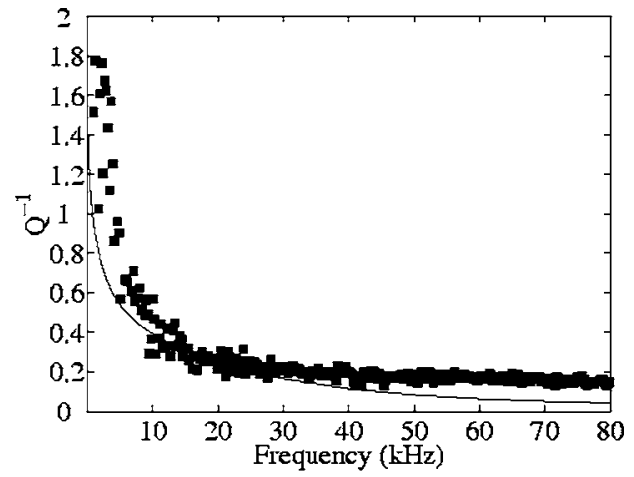

(b)
FIG. 7. Frequency-dependent attenuation coefficients of the pseudoStoneley wave in the sandstone confined reservoir. The experimental results are shown in dots and the theoretical calculations based on Biot's theory as solid lines. The results obtained in the modified shock tube configuration are depicted in (b). For comparison, previous measurements are shown (a). 


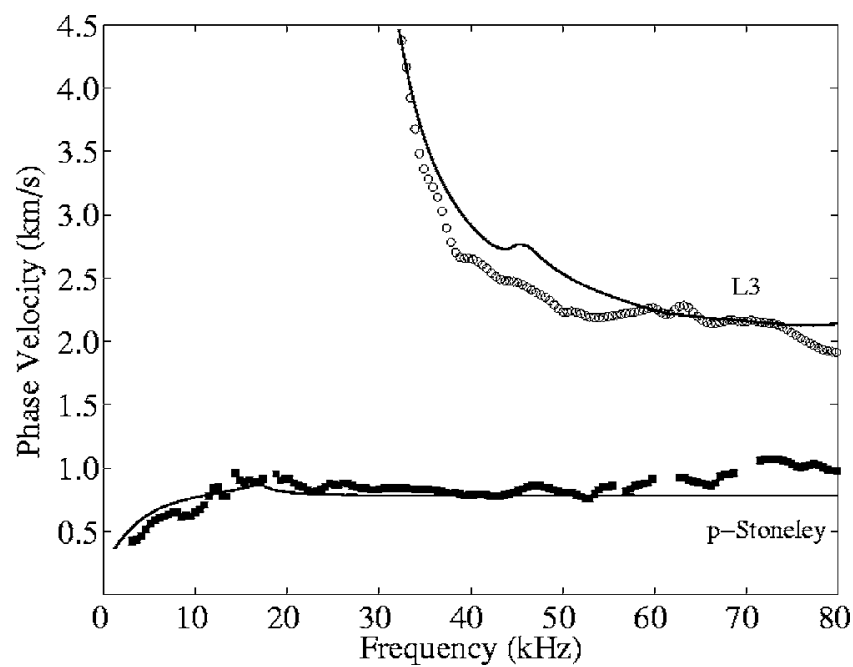

FIG. 8. Frequency-dependent phase velocities for the pseudo-Stoneley wave and the L3 mode in the synthetic N1 sample. The numerical calculations based on Biot's theory are displayed as solid lines.

isolate the wave forms corresponding to each mode in the time domain and therefore it was not feasible to apply the spectral ratio method, the attenuation data showed intense scatter (data not shown).

Finally we discuss the experimental data obtained in the N2 sample. In this case only one mode was detected and therefore the full FFT-Prony-spectral ratio method technique could be used. This mode is associated with the fast compressional wave and according to the notation introduced in Sec. II it is denoted L2. The pseudo-Stoneley wave is not observed, presumably due to the high permeability of the porous sample which implies that the attenuation of this wave is such that its amplitude is significantly damped and therefore not observed along the distances covered in the measurements. Figure 9 depicts the outcome of the experiments and the comparison with the numerical results for the L2 mode in the experimental configuration (Fig. 1). In Fig. 9 also the fast compressional wave in an unbound medium is shown in dashed lines. For this sample the correlation between the L2 mode and the fast $P$ wave extends to the complete range of frequencies of interest $(1-160 \mathrm{kHz})$. The agreement between the experimental data and the theoretical predictions for the wave velocity is fair with an overestimation of the theory at low frequencies and some underestimation at high frequencies. The attenuation of the L2 mode reveals the presence of regular oscillatory patterns. These oscillations are associated with radial resonances in the cylindrical cavity determined by the shock tube. Remarkably, the experimental attenuation data show regularly spaced maxima and minima which correspond to the oscillations predicted theoretically for the L2 mode. As an overall conclusion we remark that the attenuation measured is one order of magnitude larger than the values predicted numerically.

\section{CONCLUSIONS}

A shock wave technique was developed to study the frequency-dependent properties of the guided waves that propagate along a cylindrical liquid/porous interface in a

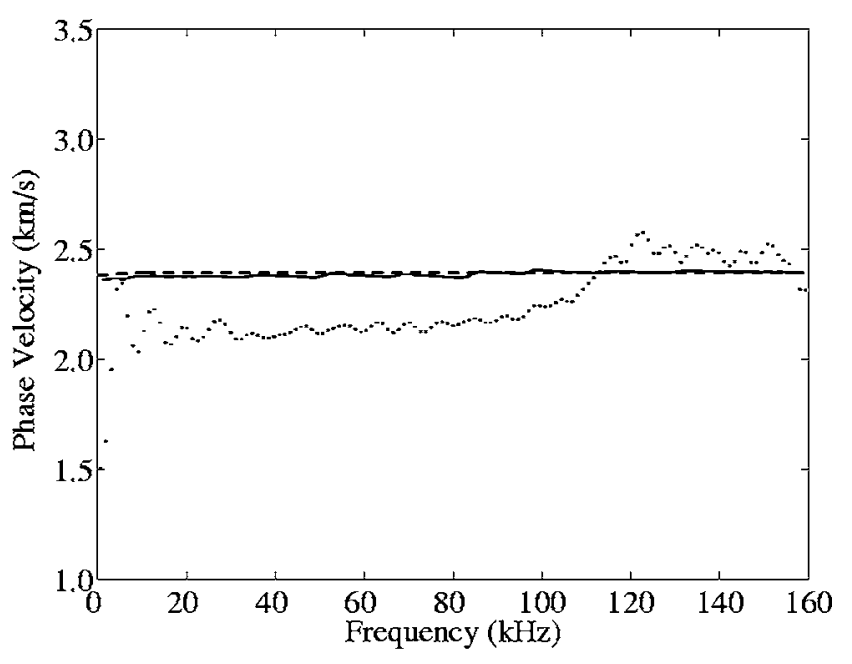

(a)

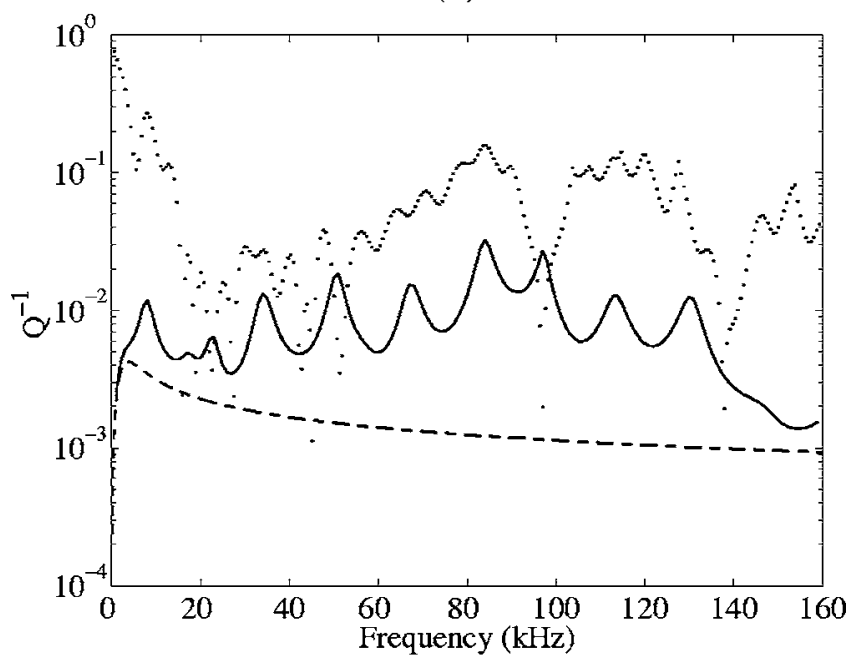

(b)

FIG. 9. Experimentally obtained velocities and specific attenuation coefficients for the L2 mode (fast P wave) in the N2 reservoir. In solid lines the numerical results for the L2 mode are shown. Biot's predictions for the velocities and attenuation coefficients of the fast compressional wave in an unbounded medium are displayed as dashed lines.

broad band of frequencies $(0.5-160 \mathrm{kHz})$. We were able to excite the pseudo-Stoneley wave in a Berea sandstone material over a broad band of frequencies. The results for the phase velocity and intrinsic attenuation agree favorably with numerical calculations based on Biot's theory. We note that using this technique we obtain experimental data at frequencies as low as $750 \mathrm{~Hz}$ which is a major improvement with respect to the experimental data available in literature. ${ }^{8,11}$ The comparison between the numerical results based on Biot's theory and the outcome of our experiments allows us to draw some conclusions on the attenuation mechanisms governing the different waves. It appears obvious that the fluid-exchange interaction at the borehole wall which is the principal loss mechanism for the pseudo-Stoneley wave is properly described by Biot's theory. Mesoscopic or microscopic dissipative mechanisms turned out to be significant at high frequencies and may be responsible for an increase ob- 
served in the experimental data with respect to the theoretical predictions, indicating a significant sensitivity of the attenuation of the pseudo-Stoneley wave on the microstructure of the porous material.

Other guided modes were also measured. The L2 mode is associated with the fast compressional wave in the porous material. Measurements of this wave performed in a highly permeable sample revealed an underestimation of the model predictions to describe the level of attenuation observed in the experimental data.

The outcome of this study is of significance in a broad band of problems related to exploration methods, reservoir characterization, and nondestructive testing. The study of the propagation of the borehole modes under well-defined laboratory conditions removes several of the uncertainties encountered in field measurements. In this sense our experimental technique can be a tool in the validation of theoretical models, inversion algorithms, and interpretation of seismic and borehole acoustic data.

\section{ACKNOWLEDGMENTS}

This study was financially supported by the ISES (Integrated Solid Earth Sciences) program. The authors acknowledge Jan Willems and Ad Holten for technical support and Robin van Gastel and Herman Koolmees for assistance during the experiments.

${ }^{1}$ Z. E. A. Fellah, F. G. Mitri, C. Depollier, S. Berger, W. Lauriks, and J. Y. Chapelon, J. Appl. Phys. 94, 7914 (2003).

${ }^{2}$ Q. Fan, J. Takatsubo, and S. Yamamoto, J. Appl. Phys. 86, 4023 (1999).

${ }^{3}$ J. F. Allard, M. Henry, C. Glorieux, S. Petillon, and W. Lauriks, J. Appl.
Phys. 93, 1298 (2003).

${ }^{4}$ J. F. Allard, M. Henry, C. Glorieux, W. Lauriks, and S. Petillon, J. Appl. Phys. 95, 528 (2004).

${ }^{5}$ L. Boeckx, P. Leclaire, P. Khurana, C. Glorieux, W. Lauriks, and J. F. Allard, J. Appl. Phys. 97, 094911 (2005).

${ }^{6}$ C. H. Cheng, M. N. Toksoz, and M. E. Willis, J. Geophys. Res. B 87, 5477 (1982).

${ }^{7}$ D. P. Schmitt, M. Bouchon, and G. Bonnet, Geophysics 53, 807 (1988).

${ }^{8}$ K. W. Winkler, H.-S. Liu, and D. L. Johnson, Geophysics 54, 66 (1989).

${ }^{9}$ A. N. Norris, Geophysics 54, 330 (1989).

${ }^{10}$ X. M. Tang, C. H. Cheng, and M. N. Toksöz, J. Acoust. Soc. Am. 90, 1632 (1991).

${ }^{11}$ G. Chao, D. M. J. Smeulders, and M. E. H. van Dongen, J. Acoust. Soc. Am. 116, 693 (2004).

${ }^{12}$ S. R. Pride, J. G. Berryman, and J. M. Harris, J. Geophys. Res. 109, B01201 (2004).

${ }^{13}$ M. A. Biot, J. Acoust. Soc. Am. 28, 168 (1956).

${ }^{14}$ M. A. Biot, J. Acoust. Soc. Am. 28, 179 (1956).

${ }^{15}$ M. A. Biot, J. Appl. Phys. 33, 1482 (1962).

${ }^{16}$ S. R. Pride and J. G. Berryman, Phys. Rev. E 68, 036603 (2003).

${ }^{17}$ S. R. Pride and J. G. Berryman, Phys. Rev. E 68, 036604 (2003).

${ }^{18}$ J. G. van der Grinten, M. E. H. van Dongen, and H. van der Kogel, J. Appl. Phys. 58, 2937 (1985).

${ }^{19}$ J. G. van der Grinten, M. E. H. van Dongen, and H. van der Kogel, J. Appl. Phys. 62, 4682 (1987).

${ }^{20}$ D. M. J. Smeulders and M. E. H. van Dongen, J. Fluid Mech. 343, 351 (1997).

${ }^{21}$ B. K. Sinha and S. Asvadurov, Geophys. Prospect. 52, 271 (2004).

${ }^{22}$ S. Feng and D. L. Johnson, J. Acoust. Soc. Am. 74, 906 (1983).

${ }^{23}$ L. D. Lafleur and F. D. Shields, J. Acoust. Soc. Am. 97, 1435 (1995).

${ }^{24}$ B. K. Sinha, T. J. Plona, S. Kostek, and S. K. Chong, J. Acoust. Soc. Am. 92, 1132 (1992)

${ }^{25}$ C. J. Wisse, D. M. J. Smeulders, M. E. H. van Dongen, and G. Chao, J. Acoust. Soc. Am. 112, 890 (2002).

${ }^{26}$ S. L. Marple, Digital Spectral Analysis: With Applications (Prentice-Hall, Englewood Cliffs, 1987).

${ }^{27}$ N. N. Toksoz, D. H. Johnston, and A. Timur, Geophysics 44, 681 (1979). 